\title{
PERCEPCIÓN COMUNITARIA DE LA RESPONSABILIDAD \\ SOCIAL EMPRESARIAL EN OCHO DISTRITOS \\ DE LA CIUDAD DE AREQUIPA
}

\section{COMMUNITY PERCEPTION OF CORPORATE SOCIAL RESPONSIBILITY IN EIGHT DISTRICTS FROM AREQUIPA CITY}

\begin{abstract}
Walter Arias Gallegos, Leslie Chambi Tueros, Luis Torres Rodríguez, Alexander Ruelas Coronel, Max Concha Chirinos, Lizeth Huayta Chambi, Maykely Valencia Ferro, Francis Chávez Reynoso, Carlos Rivera Oviedo y Eduardo Lazo Tarqui Universidad Católica San Pablo, Arequipa, Perú
\end{abstract}

\section{Resumen:}

En el presente artículo se compara la percepción que tienen los pobladores de ocho distritos de Arequipa sobre la responsabilidad social empresarial. Para ello se tomó una muestra de 350 personas de la ciudad de Arequipa y se aplicó un instrumento validado para tales fines, que cuenta con una estructura factorial de tres componentes: beneficios sociales de la RSE, beneficios individuales de la RSE y aspectos medio ambientales de la RSE. Los resultados indican que los aspectos medio ambientales son mejor valorados por la comunidad, seguidos de los beneficios sociales y de los beneficios individuales, que son los peor valorados.

Palabras clave: Responsabilidad social empresarial, percepción comunitaria, Arequipa.

\section{Summary:}

This article compares the perception of habitants from eight districts in Arequipa about corporate social responsibility. For that, we took a sample of 350 people from Arequipa City and we applied an inventory with three factors: social benefits, individual 
benefits and environmental topics. The results indicated that environmental topics are the best valued by the community, followed by the social and individual benefits which are worst valued.

Key words: Corporate social responsibility, community perception, Arequipa.

Hoy en día las empresas juegan un papel relevante para combatir la pobreza y promover el desarrollo de las comunidades porque concentran poder económico y cuentan con recursos para transformar favorablemente su entorno (Del Castillo y Bose, 2008). En esa medida, la responsabilidad social empresarial (RSE) sirve de marco para orientar y comprender cómo es que se producen relaciones entre la empresa, la comunidad y el Estado; con la finalidad de sumar esfuerzos para mejorar la calidad de vida de los pobladores de una comunidad determinada.

Entre los factores que hicieron posible su surgimiento se tiene el aumento de la sensibilidad social, el nuevo sentido de integración, la búsqueda de calidad laboral, la aparición del concepto de capital social, la necesidad de vencer la desigualdad social, el concepto de desarrollo sostenible, la necesidad de la participación social y la consecuente modificación de concepto de empresa (Vélez, 2010). Podemos decir que la RSE surge en EE.UU. como respuesta al debate sobre las exigencias y necesidades de un mundo globalizado, particularmente sobre el tema social y medioambiental (Castaño, 2012).

En ese sentido, históricamente, las primeras nociones de empresa se sustentaban en una ética utilitarista, para luego seguir criterios racionales orientados a la productividad y la eficiencia (Correa, 2007). Friedman por ejemplo, señalaba que en una economía libre, la única responsabilidad social en los negocios debía ser la de generar mayores ganancias y preservar el sistema libre competencia (Giménez, Reyes y Gómez, 2007). Hoy en día se busca que las empresas exhiban un comportamiento ético genuino (Manzone, 2007), vale decir que las empresas no deben ser juzgadas solo por sus resultados, sino por su comportamiento ante la sociedad (Cejas y Camejo, 2009).

Sin embargo, en países como el Perú, todavía nos regimos por valores utilitarios de manera predominante, lo cual dificulta la adopción de un sistema de responsabilidad social como ocurre en países que tienen ya larga data en la inserción a los mercados globales. Por otro lado, existen diversas maneras de medir la responsabilidad social y de concebirla (Giménez, Reyes y Gómez, 2007), lo cual hace más difícil aún su asunción en el escenario local y nacional. Además, la historia y la identidad de la empresa son factores clave para que se pueda desarrollar en su seno un sistema de responsabilidad social (Castaño, 2012).

Por otro lado, aunque con el correr del tiempo, la RSE se ha convertido en una obligación legal, no puede considerarse como verdadera si no nace de manera espontánea desde la propia identidad de la empresa. En ese sentido, las empresas socialmente responsables asumen una actitud proactiva no reactiva, y se caracterizan por el liderazgo que adquieren frente a sus rivales (Trujillo y Vélez, 2006), vale decir que no actúan favorablemente en la sociedad por compromiso sino por convicción, lo que 
supone no solo hacer actividades mediáticas en beneficio de la comunidad (colectas, donativos, etc.), sino desarrollar todo un plan de acción, que apuntando a diversos actores (stakeholders), se promueva la transformación social, económica y material de la comunidad en la que operan las empresas. Esto supone valorar la relación entre la empresa y la sociedad, los trabajadores y el medio ambiente (Vélez, 2010).

En consecuencia, la RSE se define como la capacidad de respuesta que tiene una empresa frente a las implicancias de sus acciones sobre los diferentes grupos de interés con los que se relaciona (Castaño, 2012). Así pues las empresas no pueden verse solamente como productoras de bienes y servicios, ya que no se trata solo de obtener utilidades sino de contribuir con aquellos que participan en las empresas (Barroso, 2008). Por ello, la RSE significa un cambio en el paradigma de los intereses de la empresa, ya que no solo ve los resultados a corto plazo, sino el conjunto de los actores sociales (Roitstein, 2004), de modo que la ética y los valores son las directrices de la actividad de la empresa.

Para la Comisión de Comunidades Europeas la empresa socialmente responsable es aquella cuyos productos y servicios contribuyen al bienestar social, sus directivos tienen un comportamiento ético, su desarrollo contempla el apoyo a los menos favorecidos y su comportamiento va más allá del estricto cumplimiento de la norma (Núñez y Alonso, 2006). En ese sentido, toda empresa tiene responsabilidad social, porque en todos los casos se debe incrementar la calidad de vida del ser humano, independientemente del rubro en que se trabaje, o de los actores con los que se relacione (Zarco y Rodríguez, 2005). Sin embargo, no toda empresa es socialmente responsable, ya que es muy común ver que las empresas de Arequipa se preocupan muy poco por sus trabajadores: no les provee de ambientes y recursos materiales adecuados para trabajar, están sujetos a diversas variables de riesgo psicosocial y las relaciones humanas se encuentran bastante deterioradas (Arias, 2013; Arias Jiménez, 2012; Arias y Jiménez, 2013).

Con respecto al medio ambiente, no hay un compromiso firme, por preservarlo o de cuidar el ecosistema, a pesar de que el tratado de Kyoto se firmó hace ya casi 20 años atrás (Franco, Chang, León y Mendoza, 2009). Tampoco hay un interés cabal por contribuir con la sociedad, ya que solo las grandes empresas del sector de la minería ejecutan obras de desarrollo económico y social en la ciudad. Esto es así, porque los principios de la responsabilidad social no se han difundido lo suficiente entre las empresas del Perú, y mucho menos entre la población. Es decir que las empresas desconocen que la idea principal de la RSE es convertirse en un factor estratégico de desarrollo y de competitividad en función de la rentabilidad que producen. Así pues se ignora que las empresas que ponen en práctica la RSE se hacen más competitivas, porque se convierten en foco de desarrollo para la comunidad, lo cual les brinda un mejor posicionamiento en el mercado.

Por ello, es que se debe promover la RSE como política general de las empresas, y establecer un sistema de gestión de responsabilidad social corporativa, que difunda adecuadamente la información sobre sus actuaciones en las cuestiones antes señaladas (Núñez y Alonso, 20o6). Ya que si no comunican sus acciones, la comunidad no 
se da por enterada. En tal sentido, no solo se debe buscar beneficiar a más personas de la comunidad, sino comunicarlo ampliamente (Castaño, 2012). La presente investigación intenta dar a conocer cómo son percibidas las empresas de la ciudad de Arequipa, en función de los criterios de la responsabilidad social. Para lo cual se ha diseñado un estudio comparativo, tomando como criterio de comparación a ocho distritos de Arequipa metropolitana.

\section{Método}

\section{Tipo y diseño de investigación}

Se trata de un estudio comparativo de tipo cuantitativo, que emplea un diseño de investi- gación descriptivo transeccional (Hernández, Fernández y Baptista, 2006).

\section{Muestra}

La muestra está constituida por 350 personas, varones y mujeres mayores de edad, que provienen de ocho distritos de la ciudad de Arequipa. En la Tabla 1 se puede apreciar cómo está distribuida la muestra por distritos. La muestra fue seleccionada por medio de la técnica de sujetos voluntarios, por tanto se trata de un muestreo no probabilístico, pero que representan proporcionalmente, aunque no significativamente, la población del distrito del que provienen. Como criterio de inclusión se consideró que los sujetos vivieran en los distritos que forman parte de la muestra.

Tabla 1.

Distribución de la muestra por distritos

\begin{tabular}{|l|c|c|c|}
\hline & Frecuencia & Porcentaje & Porcentaje acumulado \\
\hline Yanahuara & 40 & 11.428 & 11.428 \\
\hline Cerro Colorado & 51 & 14.571 & 26 \\
\hline Miraflores & 39 & 11.142 & 37.142 \\
\hline JLByR & 40 & 11.428 & 48.571 \\
\hline ASA & 40 & 11.428 & 60 \\
\hline Cayma & 40 & 11.428 & 71.428 \\
\hline Socabaya & 40 & 11.428 & 82.857 \\
\hline Paucarpata & 60 & 17.142 & 100 \\
\hline Total & 350 & 100 & \\
\hline
\end{tabular}

\section{Instrumentos}

Como instrumento se utilizó un inventario que mide la percepción comunitaria de la responsabilidad social empresarial, que consta de 44 ítems dispuestos en una escala tipo Likert, y que cuenta con validez de conte- nido a través del juicio de expertos, presenta homogeneidad en tanto que las correlaciones ítem test son superiores a 0.20 y se ubican en un rango de 0.221 a 0.567 . También cuenta con una estructura factorial de tres componentes: beneficios de la responsabilidad a nivel local $(\alpha=0.866)$, beneficios 
individuales de la responsabilidad social $(\alpha=0.802)$ y aspectos medio ambientales $(\alpha=0.739)$ con índices alfa de Cronbach superiores a o.70. Por tanto se trata de un instrumento válido y confiable. Mayores datos sobre el proceso de elaboración de la prueba se pueden encontrar en Arias, Dominguez, Docarmo, Raschio, Rojas, Gutiérrez y Condori (en revisión).

\section{Procedimiento}

Los datos fueron recogidos durante los meses de agosto y diciembre del año 2012, por miembros del equipo de investigación, que está conformado por estudiantes de los programas de contabilidad y administración de empresas de la Universidad Católica San Pablo. Las personas que componen la muestra fueron abordadas en los respectivos distritos que fueron considerados en este estudio, y por medio de la técnica de la entrevista se les aplicó el inventario. Los datos se recogieron en lugares públicos como plazas, parques o avenidas de alto tránsito en los ocho distritos seleccionados, durante horas del día hasta la media tarde.

\section{Resultados}

En primer lugar se presentan los datos descriptivos de las dimensiones de la percepción comunitaria de la responsabilidad social empresarial en la Tabla 2. La media obtenida en la dimensión beneficios sociales es de 55.005 lo que la ubica en un nivel promedio, la media para beneficios individuales es 36.188 lo que también le ubica en un nivel promedio, al igual que la dimensión aspectos medio ambientales, que tiene una media de 27.368. Las desviaciones estándar son bajas y la asimetría indica que los valores tienden a ser favorables porque se ubican hacia la derecha, aunque muy levemente.

Tabla 2.

Valores descriptivos de la percepción comunitaria de la RSE

\begin{tabular}{|l|c|c|c|c|}
\hline & $\begin{array}{c}\text { Beneficios } \\
\text { sociales }\end{array}$ & $\begin{array}{c}\text { Beneficios } \\
\text { individuales }\end{array}$ & $\begin{array}{c}\text { Aspectos medio } \\
\text { ambientales }\end{array}$ & Total \\
\hline Media & 55.005 & 36.188 & 27.368 & 116.142 \\
\hline Mediana & 56 & 36 & 27 & 117 \\
\hline Moda & 60 & 35 & 26 & 121 \\
\hline Desviación típica & 8.539 & 5.960 & 4.568 & 14.755 \\
\hline Varianza & 72.916 & 35.523 & 20.869 & 217.721 \\
\hline Asimetría & -0.143 & 0.122 & -0.011 & -0.182 \\
\hline Curtosis & -0.004 & 0.864 & 0.202 & 0.473 \\
\hline Mínimo & 28 & 19 & 13 & 70 \\
\hline Máximo & 75 & 59 & 42 & 167 \\
\hline
\end{tabular}


Haciendo un análisis de la distribución de porcentajes (Tabla 3), tenemos que del total de la muestra el $52.857 \%$ se ubica en el nivel medio, el $24.857 \%$ en el nivel bajo y el $22.285 \%$ en el nivel alto, con respecto a la dimensión de beneficios sociales de la responsabilidad social empresarial percibida por la comunidad. Los beneficios individuales son percibidos en su mayoría ( $46.857 \%$ ) en un nivel medio, seguidos del nivel bajo (31.142\%) y del nivel alto (22\%). Lo que implica la percepción comunitaria de los beneficios individuales de la responsabilidad social empresarial es más negativa que los beneficios sociales. Lo contrario ocurre con los aspectos medio ambientales que son valorados más favorablemente que las dos dimensiones anteriores, pues el $52.857 \%$ los valora en un nivel promedio, $40.571 \%$ en un nivel alto y solo el $6.571 \%$ en un nivel bajo.

Tabla 3.

Porcentajes según el nivel de RSE por factores

\begin{tabular}{|l|l|r|r|}
\hline & \multicolumn{1}{|c|}{ Nivel } & \multicolumn{1}{|c|}{ Frecuencia } & \multicolumn{1}{c|}{ Porcentaje } \\
\hline \multirow{4}{*}{$\begin{array}{l}\text { Beneficios sociales de } \\
\text { la RSE }\end{array}$} & Bajo & 87 & 24.857 \\
\cline { 2 - 4 } & Medio & 185 & 52.857 \\
\cline { 2 - 4 } & Alto & 78 & 22.285 \\
\hline \multirow{4}{*}{$\begin{array}{l}\text { Beneficios individuales } \\
\text { RSE }\end{array}$} & Bajo & 109 & 31.142 \\
\cline { 2 - 4 } & Medio & 164 & 46.857 \\
\cline { 2 - 4 } & Alto & 77 & 22 \\
\hline \multirow{3}{*}{$\begin{array}{l}\text { Aspectos medio } \\
\text { ambientales de la RSE }\end{array}$} & Bajo & 23 & 6.571 \\
\cline { 2 - 4 } & Medio & 185 & 52.857 \\
\cline { 2 - 4 } & Alto & 142 & 40.571 \\
\hline
\end{tabular}

En la Tabla 4 se observan los valores de las correlaciones de los tres factores con el puntaje total, donde se tiene que los tres factores se relacionan alta y significativamente con el puntaje total de la prueba, lo que da cuenta de las propiedades psicométricas del instrumento. Sin embargo es interesante notar que los beneficios sociales se relacionan de manera baja moderada con los beneficios individuales $(r=0.375)$, pero que se relacionan de manera más alta con los aspectos medio ambientales $(\mathrm{r}=0.513)$, mientras que los beneficios individuales se relacionan en menor medida con los aspectos medio ambientales $(\mathrm{r}=0.397)$. Esto sugiere que la población percibe que la responsabilidad social de las empresas tiene un impacto más alto en la colectividad que en las personas como individuos, y que los aspectos medio ambientales nos benefician individualmente. 
Tabla 4 .

Correlaciones entre dimensiones

\begin{tabular}{|c|c|c|c|c|}
\hline & $\begin{array}{c}\text { Beneficios } \\
\text { sociales }\end{array}$ & $\begin{array}{l}\text { Beneficios } \\
\text { individuales }\end{array}$ & $\begin{array}{l}\text { Aspectos medio } \\
\text { ambientales }\end{array}$ & Total \\
\hline $\begin{array}{l}\text { Beneficios } \\
\text { sociales }\end{array}$ & 1 & 0.375 & 0.397 & 0.847 \\
\hline $\begin{array}{l}\text { Beneficios } \\
\text { individuales }\end{array}$ & & 1 & 0.513 & 0.755 \\
\hline $\begin{array}{l}\text { Aspectos medio } \\
\text { ambientales }\end{array}$ & & & 1 & 0.721 \\
\hline Total & & & & 1 \\
\hline
\end{tabular}

* Todos los valores son significativos al $\mathrm{p}<0.000$

También realizamos un análisis por distritos sabilidad social empresarial, para lo cual se con respecto a cada una de las dimensiones elaboraron tablas de contingencia y se valoró de la percepción comunitaria de la respon- la significancia con la prueba chi cuadrado.

Tabla 5.

Tabla de contingencia de los Beneficios sociales de la RSE por distrito

\begin{tabular}{|c|c|c|c|c|c|}
\hline & & \multicolumn{3}{|c|}{ Beneficios sociales } & \multirow{2}{*}{ Total } \\
\hline & & Bajo & Medio & Alto & \\
\hline \multirow{4}{*}{ Yanahuara } & Recuento & 4 & 12 & 24 & 40 \\
\hline & $\%$ de Distrito & 10 & 30 & 60 & 100 \\
\hline & $\%$ de Beneficios sociales & $4 \cdot 597$ & 6.486 & 30.769 & 11.428 \\
\hline & $\%$ del total & 1.142 & 3.428 & 6.857 & 11.428 \\
\hline \multirow{4}{*}{ Cerro Colorado } & Recuento & o & 36 & 15 & 51 \\
\hline & $\%$ de Distrito & o & 70.588 & 29.411 & 100 \\
\hline & $\%$ de Beneficios sociales & o & 19.459 & 19.230 & 14.571 \\
\hline & $\%$ del total & o & 10.285 & 4.285 & 14.571 \\
\hline \multirow{4}{*}{ Miraflores } & Recuento & 4 & 27 & 8 & 39 \\
\hline & $\%$ de Distrito & 10.256 & 69.230 & 20.512 & 100 \\
\hline & $\%$ de Beneficios sociales & $4 \cdot 597$ & 14.594 & 10.256 & 11.142 \\
\hline & $\%$ del total & 1.142 & $7 \cdot 714$ & 2.285 & 11.142 \\
\hline
\end{tabular}




\begin{tabular}{|c|c|c|c|c|c|}
\hline \multirow{4}{*}{ JLByR } & Recuento & 35 & 5 & o & 40 \\
\hline & $\%$ de Distrito & 87.5 & 12.5 & o & 100 \\
\hline & $\%$ de Beneficios sociales & 40.229 & 2.702 & o & 11.428 \\
\hline & $\%$ del total & 10 & 1.428 & o & 11.428 \\
\hline \multirow{4}{*}{ ASA } & Recuento & 22 & 18 & o & 40 \\
\hline & $\%$ de Distrito & 55 & 45 & o & 100 \\
\hline & \% de Beneficios sociales & 25.287 & 9.729 & o & 11.428 \\
\hline & $\%$ del total & 6.285 & 5.142 & o & 11.428 \\
\hline \multirow{4}{*}{ Cayma } & Recuento & 3 & 27 & 10 & 40 \\
\hline & $\%$ de Distrito & $7 \cdot 5$ & 67.5 & 25 & 100 \\
\hline & $\%$ de Beneficios sociales & 3.448 & 14.594 & 12.820 & 11.428 \\
\hline & $\%$ del total & 0.857 & $7 \cdot 714$ & 2.857 & 11.428 \\
\hline \multirow{4}{*}{ Socabaya } & Recuento & 5 & 19 & 16 & 40 \\
\hline & $\%$ de Distrito & 12.5 & $47 \cdot 5$ & 40 & 100 \\
\hline & $\%$ de Beneficios sociales & $5 \cdot 747$ & 10.270 & 20.512 & 11.428 \\
\hline & $\%$ del total & 1.428 & $5 \cdot 428$ & $4 \cdot 571$ & 11.428 \\
\hline \multirow{4}{*}{ Paucarpata } & Recuento & 14 & 41 & 5 & 60 \\
\hline & $\%$ de Distrito & 23.333 & 68.333 & 8.333 & 100 \\
\hline & $\%$ de Beneficios sociales & 16.091 & 22.162 & 6.410 & 17.142 \\
\hline & $\%$ del total & 4 & 11.714 & 1.428 & 17.142 \\
\hline
\end{tabular}

$\mathrm{X}^{2}=186.6 ; \mathrm{gl}=14 ; \mathrm{p}<0.001$

En la Tabla 5 se observa que en distritos como Yanahuara la percepción de los beneficios sociales de la responsabilidad social empresarial es más alta que en todos los demás distritos, mientras que en José Luis Bustamante y Rivero y Alto Selva Alegre, el porcentaje de gente que obtiene un nivel bajo es mayor, al punto de no tener nadie que llega a un nivel alto. En Socabaya, Cayma, Miraflores, y Cerro Colorado, los beneficios sociales se perciben favorablemente, mientras que en Paucarpata se perciben de manera más desfavorable. 
Tabla 6.

Tabla de contingencia de los Beneficios individuales de la RSE por distrito

\begin{tabular}{|c|c|c|c|c|c|}
\hline & & \multicolumn{3}{|c|}{ Beneficios individuales } & \multirow{2}{*}{ Total } \\
\hline & & Bajo & Medio & Alto & \\
\hline \multirow[t]{4}{*}{ Yanahuara } & Recuento & 11 & 28 & 1 & 40 \\
\hline & $\%$ de Distrito & 27.5 & 70 & 2.5 & 100 \\
\hline & \% de B. individuales & 10.091 & 17.073 & 1.298 & 11.428 \\
\hline & $\%$ del total & 3.142 & 8 & 0.285 & 11.428 \\
\hline \multirow[t]{4}{*}{ Cerro Colorado } & Recuento & 49 & 2 & o & 51 \\
\hline & $\%$ de Distrito & 96.078 & 3.921 & o & 100 \\
\hline & \% de B. individuales & 44.954 & 1.219 & $\mathrm{o}$ & 14.571 \\
\hline & $\%$ del total & 14 & 0.571 & o & 14.571 \\
\hline \multirow[t]{4}{*}{ Miraflores } & Recuento & 13 & 19 & 7 & 39 \\
\hline & \% de Distrito & $33 \cdot 333$ & 48.717 & 17.948 & 100 \\
\hline & $\%$ de B. individuales & 11.926 & 11.585 & 9.090 & 11.142 \\
\hline & $\%$ del total & 3.714 & 5.428 & 2 & 11.142 \\
\hline \multirow[t]{4}{*}{ JLByR } & Recuento & 7 & 27 & 6 & 40 \\
\hline & $\%$ de Distrito & $17 \cdot 5$ & 67.5 & 15 & 100 \\
\hline & \% de B. individuales & 6.422 & 16.463 & 7.792 & 11.428 \\
\hline & $\%$ del total & 2 & $7 \cdot 714$ & 1.714 & 11.428 \\
\hline \multirow[t]{4}{*}{ ASA } & Recuento & 12 & 21 & 7 & 40 \\
\hline & \% de Distrito & 30 & 52.5 & 17.5 & 100 \\
\hline & $\%$ de B. individuales & 11.009 & 12.804 & 9.090 & 11.428 \\
\hline & $\%$ del total & 3.428 & 6 & 2 & 11.428 \\
\hline \multirow[t]{4}{*}{ Cayma } & Recuento & 5 & 26 & 9 & 40 \\
\hline & $\%$ de Distrito & 12.5 & 65 & 22.5 & 100 \\
\hline & \% de B. individuales & 4.587 & 15.853 & 11.688 & 11.428 \\
\hline & $\%$ del total & 1.428 & $7 \cdot 428$ & 2.571 & 11.428 \\
\hline \multirow[t]{4}{*}{ Socabaya } & Recuento & 1 & 13 & 26 & 40 \\
\hline & $\%$ de Distrito & 2.5 & 32.5 & 65 & 100 \\
\hline & \% de B. individuales & 0.917 & $7 \cdot 926$ & 33.766 & 11.428 \\
\hline & $\%$ del total & 0.285 & 3.714 & $7 \cdot 428$ & 11.428 \\
\hline \multirow[t]{4}{*}{ Paucarpata } & Recuento & 11 & 28 & 21 & 60 \\
\hline & \% de Distrito & 18.333 & 46.666 & 35 & 100 \\
\hline & $\%$ de $\mathrm{B}$. individuales & 10.091 & 17.073 & 27.272 & 17.142 \\
\hline & $\%$ del total & 3.142 & 8 & 6 & 17.1428571 \\
\hline
\end{tabular}

$\mathrm{X}^{2}=180.913 ; \mathrm{gl}=14 ; \mathrm{p}<0.000$ 
En la Tabla 6 se puede ver que en el distrito de Cerro Colorado la mayoría de personas (96\%) perciben bajos beneficios individuales de la responsabilidad social empresarial. En cambio, en Yanahuara, Miraflores, José Luis Bustamante y Rivero, Alto Selva Alegre,
Cayma y Paucarpata, la mayoría de pobladores se ubica en un nivel medio de percepción de los beneficios individuales. Mientras que en Socabaya un $65 \%$ percibe los beneficios individuales de la responsabilidad social empresarial, de manera elevada.

Tabla 7.

Tabla de contingencia de los Aspectos medio ambientales de la RSE por distrito

\begin{tabular}{|c|c|c|c|c|c|}
\hline & & \multicolumn{3}{|c|}{ Aspectos medio ambientales } & \multirow{2}{*}{ Total } \\
\hline & & Bajo & Medio & Alto & \\
\hline \multirow[t]{4}{*}{ Yanahuara } & Recuento & 1 & 16 & 23 & 40 \\
\hline & $\%$ de Distrito & 2.5 & 40 & 57.5 & 100 \\
\hline & $\%$ de Medio ambiente & $4 \cdot 347$ & 8.648 & 16.197 & 11.428 \\
\hline & $\%$ del total & 0.285 & $4 \cdot 571$ & 6.571 & 11.428 \\
\hline \multirow[t]{4}{*}{ Cerro Colorado } & Recuento & o & 47 & 4 & 51 \\
\hline & $\%$ de Distrito & o & 92.156 & 7.843 & 100 \\
\hline & $\%$ de Medio ambiente & o & 25.405 & 2.816 & 14.571 \\
\hline & $\%$ del total & o & 13.428 & 1.142 & $14 \cdot 571$ \\
\hline \multirow[t]{4}{*}{ Miraflores } & Recuento & 4 & 19 & 16 & 39 \\
\hline & $\%$ de Distrito & 10.256 & 48.717 & 41.025 & 100 \\
\hline & $\%$ de Medio ambiente & 17.391 & 10.270 & 11.267 & 11.1428 \\
\hline & $\%$ del total & 1.142 & 5.428 & $4 \cdot 571$ & 11.142 \\
\hline \multirow[t]{4}{*}{ JLByR } & Recuento & 3 & 26 & 11 & 40 \\
\hline & $\%$ de Distrito & $7 \cdot 5$ & 65 & $27 \cdot 5$ & 100 \\
\hline & $\%$ de Medio ambiente & 13.043 & 14.054 & $7 \cdot 746$ & 11.428 \\
\hline & $\%$ del total & 0.857 & $7 \cdot 428$ & 3.142 & 11.428 \\
\hline \multirow[t]{4}{*}{ ASA } & Recuento & 6 & 26 & 8 & 40 \\
\hline & $\%$ de Distrito & 15 & 65 & 20 & 100 \\
\hline & $\%$ de Medio ambiente & 26.086 & 14.054 & 5.633 & 11.428 \\
\hline & $\%$ del total & 1.714 & $7 \cdot 428$ & 2.285 & 11.428 \\
\hline \multirow[t]{4}{*}{ Cayma } & Recuento & 3 & 23 & 14 & 40 \\
\hline & $\%$ de Distrito & $7 \cdot 5$ & $57 \cdot 5$ & 35 & 100 \\
\hline & $\%$ de Medio ambiente & 13.043 & 12.432 & 9.859 & 11.428 \\
\hline & $\%$ del total & 0.857 & 6.571 & 4 & 11.428 \\
\hline
\end{tabular}




\begin{tabular}{|l|l|c|c|c|c|}
\hline Socabaya & Recuento & 0 & 13 & 27 & 40 \\
\cline { 2 - 6 } & \% de Distrito & 0 & 32.5 & 67.5 & 100 \\
\cline { 2 - 6 } & \% de Medio ambiente & 0 & 7.027 & 19.014 & 11.428 \\
\cline { 2 - 6 } & \% del total & 0 & 3.714 & 7.714 & 11.428 \\
\hline Paucarpata & Recuento & 6 & 15 & 39 & 60 \\
\cline { 2 - 6 } & \% de Distrito & 10 & 25 & 65 & 100 \\
\cline { 2 - 6 } & \% de Medio ambiente & 26.086 & 8.108 & 27.464 & 17.142 \\
\cline { 2 - 6 } & \% del total & 1.714 & 4.285 & 11.142 & 17.142 \\
\hline
\end{tabular}

$\mathrm{X}^{2}=82.346 ; \mathrm{gl}=14 ; \mathrm{p}<0.05$

Finalmente, en la Tabla 7 se tiene que en distritos como Yanahuara, Socabaya y Paucarpata, la mayoría de pobladores perciben un alto nivel de responsabilidad social con respecto al cuidado del medio ambiente. Cayma, José Luis Bustamante y Rivero, Alto Selva Alegre, Miraflores y Cerro Colorado; son distritos en los que el mayor porcentaje de pobladores se ubica en un término medio con respecto a los aspectos medio ambientales de las responsabilidad social de las empresas. Un bajo porcentaje en todos los distritos percibe escasos beneficios medio ambientales de la responsabilidad social en Arequipa.

\section{Discusión}

La responsabilidad social empresarial se nos presenta como una alternativa de solución de diversos problemas sociales para los países latinoamericanos (Bull, 2006), en la medida que las empresas tienen un impacto profundo en la sociedad, las personas y la naturaleza. Aunque hoy, la ley obliga a las empresas a conducirse responsablemente, más que por el cumplimiento de las normas jurídicas, la responsabilidad social debe asumirse como un compromiso ético de las empresas (Guerra, 2007).
En ese sentido, las empresas que desean perdurar en el mercado deben ser buenos ciudadanos corporativos (Castaño, 2012). Barroso (2008) nos indica diez principios de la responsabilidad social empresarial: 1) promover una cultura de RS dentro de la empresa, 2) identificar necesidades sociales del entorno y colaborar con su solución, 3) hacer públicos los valores de la empresa, 4) vivir esquemas de liderazgo participativo, 5) promover el desarrollo humano y profesional de toda su comunidad, 6) apoyar causas sociales como parte de la estrategia de acción empresarial, 7) respetar el entorno ecológico, 8) invertir en el desarrollo de la comunidad, 9) formalizar alianzas con otras empresas para el beneficio de la comunidad, e 10) involucrar al personal con el desarrollo social.

Este decálogo, incluye diversos aspectos de la responsabilidad social, siendo uno de ellos la comunicación oportuna de los valores y logros sociales de la empresa. Este aspecto es capital dentro de la responsabilidad social, ya que es necesario que la población esté informada sobre el grado de compromiso que tienen las empresas del entorno con el desarrollo social de su comunidad. Sin embargo, la comunicación 
no solo es un proceso de transmisión de información, sino que debe promover el desarrollo de la empresa, a través de la retroalimentación (Rotstein, 2004). Por ello es importante que las empresas sepan cómo son percibidas por sus usuarios y clientes.

Nosotros hemos creado un instrumento que valora la percepción comunitaria de la responsabilidad social empresarial a través de tres dimensiones: los beneficios sociales de la RSE, los beneficios individuales de la RSE y los aspectos medio ambientales; que y al aplicarlo en ocho distritos de nuestra ciudad tenemos que los aspectos medio ambientales son mejor percibidos por la sociedad, seguidos de los beneficios sociales y en último lugar se ubican los beneficios individuales. Estos resultados, reafirman lo que varios autores han señalado, cuando advierten que el cuidado del medio ambiente es el que se identifica más con la responsabilidad social (Hamann, 2007), a pesar de no haber fundamentos objetivos que reafirmen dicha afirmación (Zicari, 2006).

Por otro lado, el hecho de que los beneficios sociales de la RSE hayan obtenido valores más altos que los beneficios individuales, podría relacionarse con la muy extendida idea de que aunque en general se perciben mejoras sustanciales en la economía del país, estas todavía no llegan a los bolsillos de la mayoría de las personas. Parece haber un notorio desarrollo económico de las empresas, la infraestructura de las ciudades, etc.; pero la economía individual está todavía muy ajustada en los sectores más populares, que corresponden a la mayoría de la población.
Asimismo es posible encontrar diferencias por distrito con respecto a la forma en que la población percibe la responsabilidad social empresarial. Mientras en distritos como José Luis Bustamante y Rivero y Alto Selva Alegre, un alto porcentaje de gente percibe que los beneficios sociales de la RSE son bajos, en Socabaya, Cayma, Miraflores y Cerro Colorado, se tiene una percepción más favorable. Para el caso de los beneficios individuales Socabaya obtiene un nivel alto, Cerro Colorado un nivel bajo y Yanahuara, Miraflores, José Luis Bustamante y Rivero, Alto Selva Alegre, Cayma y Paucarpata; un nivel medio. Los aspectos medio ambientales son mejor valorados en Yanahuara, Socabaya y Paucarpata, mientras que en Cayma, José Luis Bustamante y Rivero, Alto Selva Alegre, Miraflores y Cerro Colorado esta valoración se ubica en un nivel medio.

Es posible que en estas respuestas medien las sensaciones de bienestar de la población, que a su vez tienen que ver con el grado de satisfacción con las gestiones ediles en sus respectivos distritos, o con el nivel socioeconómico en que se ubican, ya que distritos como Yanahuara, donde se cuenta con un mejor nivel de vida, se tienen puntajes más altos en las tres dimensiones.

Por otro lado, en distritos como Socabaya y Paucarpata, con áreas campestres (campiña y zonas de cultivo) y menor contaminación, se percibe de manera favorable los aspectos medio ambientales de la responsabilidad social empresarial; lo que supone que en las respuestas vertidas pesan más otros factores, que la responsabilidad social como tal.

Evidentemente, el tema requiere de mayor indagación, pero de darse la situación que 
suponemos, existiría un divorcio entre las empresas y la sociedad, que no es exclusivo de Arequipa o el Perú, pues es muy común en Latinoamérica (Reffico y Ogliastri, 2009). En ese sentido, una posibilidad es que las empresas no se preocupan en comunicar las actividades de RSE que realizan (Méndez, 2007), o incluso peor, no implementan políticas ni actividades de RSE, lo cual les resta liderazgo en el escenario empresarial de a nivel global (Álvarez, Calderón y Rodríguez, 2011).

En consecuencia, los pobladores desconocen lo que las empresas hacen dentro del marco de la RSE porque no lo comunican eficientemente, o porque no tienen el interés en asumir las reglas vigentes del mercado que consideran el comportamiento ético de las empresas y su contribución a la sociedad y el cuidado del medio ambiente. Esto implica que nuestra sociedad no es lo suficientemente madura para tomar decisiones de mercado en función de criterios éticos y sociales como se plantea desde la teoría de la RSE, lo cual pasa de la temática de la RSE a la responsabilidad social o colectiva (Aranda y González, 2011). En ese sentido, países como Brasil, Chile o Argentina; exhiben niveles mayores de conciencia en RSE, tanto a nivel del empresariado como a nivel de la población.

Estos datos nos llevan a proponer una mayor difusión de las bondades de la RSE a nivel del empresariado arequipeño y valorar sus actitudes frente a la RSE como se ha hecho en otros países (Mababu, 2010). Además sería necesario implementar mecanismos de comunicación más eficientes por parte de las empresas locales que aplican los principios de la RSE. Aquí es necesario recordar que si bien la RSE no resuelve todos los problemas sociales, es una forma de respuesta a ellos desde la empresa (Castaño, 2012), por tanto la empresa debe comprometerse con el desarrollo de la comunidad a nivel social, individual y medio ambiental.

\section{Referencias}

Álvarez, J. L.; Calderón, R. y Rodríguez, I. (2011). Liderazgo ético y regulación en un escenario empresarial global. "Contabilidad y Negocios", 6(11), 56-66.

Aranda, J. M. y González, S. (2011). Problematización de la responsabilidad social o colectiva. “Ciencia Ergo Sum”, 18(2), 126-134.

Arias, W. L. (2013). Clima organizacional en ocho empresas de Arequipa. "Illustro", 4, 39-56.

Arias, W. L. y Jiménez, N. A. (2012). “Estrés crónico en el trabajo: Estudios sobre síndrome de burnout en Arequipa”. Arequipa: Universidad Católica San Pablo.

Arias, W. L. y Jiménez, N. A. (2013). Relaciones humanas en Mypes de Arequipa. "Contabilidad y Negocios", 8(16), 48-6o.

Arias, W. L.; Dominguez, S.; Docarmo, L.; Raschio, P.; Rojas, F.; Gutiérrez, M. C. y Condori, K. (en revisión). Presentación de un inventario para valorar la percepción comunitaria de la responsabilidad social empresarial (RSE). 
“Revista Ciencias Estratégicas” (Artículo sometido a proceso de revisión).

Barroso, F. G. (2008). La responsabilidad social empresarial. Un estudio en cuarenta empresas de la ciudad de Mérida, Yucatán. “Contaduría y Administración”, 226, 73-91.

Bull, B. (2006). Responsabilidad social de las empresas: ¿Una solución para el desarrollo de América latina? "Gerencia”, 9(28), 1-24.

Castaño, E. J. (2012). Comunicar la responsabilidad social, una opción de éxito empresarial poco explorada. "Revista Lasallista de Investigación”, 8(2), 173-186.

Cejas, M. y Camejo, A. (20o9). La dimensión estratégica de la gestión de los recursos humanos en el marco de la responsabilidad social empresarial. "Observatorio Laboral”, 2(4), 67-87.

Correa, J. G. (2007). Evolución histórica de los conceptos de responsabilidad social empresarial y balance social. “Semestre Económico", 10(20), 87-102.

Del Castillo, E. y Bose, M. (2008). Factores que influyen en la toma de decisiones sobre la RSC. "Journal of Business". Facultad de Administración y Contabilidad de la Universidad del Pacífico. (Documento en formato pdf) Recuperado el 26 de diciembre del 2013 de: https://www.up.edu.pe/carrera/ administracion/Paginas/JER/Detalle. aspx?IdElemento=75
Franco, P.; Chang, A.; León, N. y Mendoza, C. (2009). Medición financiera de la gestión de la responsabilidad social. "Journal of Business". Facultad de Administración y Contabilidad de la Universidad del Pacífico. (Documento en formato pdf) Recuperado el 4 de agosto del 2013 de: https:// www.up.edu.pe/carrera/administracion/Paginas/JER/Detalle.aspx?IdElemento $=75$

Giménez, G. B.; Reyes, J. D. y Gómez, M. (2007). La responsabilidad social en las organizaciones (RSO): Análisis y comparación entre guías y normas de gestión e información. Innovar. "Revista de Ciencias Administrativas y Sociales", 17(29), 27-47.

Guerra, A. (2007). De la responsabilidad social empresarial, a la ética en el cambio organizacional. "Compendium”, 10(18), 77-90.

Hamann, A. (2007). La empresa: actor protagónico en la gestión ambiental. “Contabilidad y Negocios”, 2(2), 47-50.

Hernández, R.; Fernández, C. y Baptista, P. (2006). "Metodología de la investigación”. México: McGraw-Hill.

Mababu, R. (2010). Actitudes de los empresarios y directivos hacia la responsabilidad social corporativa. "Revista de Psicología del Trabajo y las Organizaciones", 26(2), 101-114.

Manzone, G. (2007). "La responsabilidad de la empresa. Busines ethics y la Doctrina Social de la Iglesia en 
diálogo". Arequipa: Universidad Católica San Pablo.

Méndez, F. B. (2007). La comunicación organizacional como gestora del conocimiento y la responsabilidad social. "Razón y Palabra", 58(12), Instituto Tecnológico de Estudios Superiores de Monterrey.

Núñez, M.yAlonso, I. (2006). Propuesta deindicadores de responsabilidad social preventiva. “Universo Contábil", 2(1), 90-102.

Reficco, E. y Ogliastri, E. (2009). Empresa y sociedad en América Latina: una introducción. "Revista Latinoamericana de Administración”, 43, 1-25.

Roitstein, F. (2004). La responsabilidad social empresarial en Argentina. "Revista Latinoamericana de Administración", 32, 5-28.
Trujillo, M. A. y Vélez, R. (2006). Responsabilidad ambiental como estrategia para la perdurabilidad empresarial. "Universidad \& Empresa", 5(10), 291-308.

Vélez, A. M. (2010). Una respuesta a la acción social, de la rentabilidad a la responsabilidad social empresarial. "Revista Ciencias Estratégicas", 18(23), 11-25.

Zarco, V. y Rodríguez, A. (2005). Ética y responsabilidad social en las organizaciones actuales. "Revista Internacional de Ciencias Sociales y Humanidades SOCIOTAM", 15(2), 185-203.

Zicari, A. (2006). Responsabilidad social empresarial: del dicho al hecho. Poniéndole números a la responsabilidad social. "Invenio", 9(17), 95-105. 